\title{
Simulation with Python on transverse modes of the symmetric confocal resonator
}

\author{
Qing Hua Wang, Jing Qi, Yun Jing Ji, Yang Song, \\ Zhenhua Li
}

Qing Hua Wang, Jing Qi, Yun Jing Ji, Yang Song, Zhenhua Li, "Simulation with Python on transverse modes of the symmetric confocal resonator," Proc. SPIE 10452, 14th Conference on Education and Training in Optics and Photonics: ETOP 2017, $104525 U$ (16 August 2017); doi: 10.1117/12.2269884

SDIE Event: 14th Conference on Education and Training in Optics and Photonics, ETOP 2017, 2017, Hangzhou, China 


\title{
Simulation with Python on transverse modes of the symmetric confocal resonator
}

\author{
Wang Qing Hua*, QI Jing, Ji Yun Jing, Song Yang, Li Zhenhua \\ School of Science, Nanjing University of Science and Technology, Nanjing 210094
}

\begin{abstract}
Python is a popular open-source programming language that can be used to simulate various optical phenomena. We have developed a suite of programs to help teach the course of laser principle. The complicated transverse modes of the symmetric confocal resonator can be visualized in personal computers, which is significant to help the students understand the pattern distribution of laser resonator.
\end{abstract}

Keywords: laser resonator, transverse modes, Python, simulation

\section{INTRODUCTION}

The symmetric confocal resonators are classic stable optical resonators with many unique advantages such as small diffraction loss and simple structures ${ }^{[1]}$. A pair of quadrate mirrors or a pair of round ones can be used to constitute the most simple and common symmetric confocal resonator. The transverse optical field distributions (denoted as TEM modes following from the electromagnetic notation system) are the main factors to determine the spatial coherence and directivity of Laser, which are also the emphasis and difficulty in the learning of Laser principles. Understanding of TEM modes for both low and high orders is very useful to Laser device selection and resonator adjustment. However because of the space limitation of the textbooks, only some lower order's diffraction patterns for quadrate and round mirrors are given, many students feel TEM modes of Laser too abstract and difficult to understand.

We simulated the TEM modes for quadrate and round mirrors respectively with the Python programming language. Python is a clear and powerful object-oriented programming language and provides many user-friendly and efficient numerical routines such as routines for Hermite and Laguerre polynomials, which makes the simulation of TEM modes for Hermite-Gaussian and Laguerre-Gaussian beam more convenient ${ }^{[2,3]}$. The complicated transverse modes of the symmetric confocal resonator can be visualized in personal computers, which is significant to help the students understand the pattern distribution of laser resonator.

\section{THE THEORY FOR TRANSVERSE MODES}

During the Laser formation, a stable optical field after enough transmission of light is reached in which the relative field distribution does not vary from transit to transit and the self-reproduction appears ${ }^{[4]}$. This steady-state field distribution is usually called as the transverse mode of the open cavity. These transverse modes are essentially determinate by the Maxwell equations and boundary conditions of the resonator cavity. Given the structure of the resonator cavity, the characteristics of transverse modes are determined.

To a symmetric confocal resonator with cavity length $L$, Laser wavelength $\lambda$, and the size of $2 a \times 2 a$ quadrate mirror, the optical field near the center of mirror $(x<<a, y<<a)$ can be written as the product of Hermite polynomial and Gaussian distribution function ${ }^{[5]}$ :

$$
U_{m n}(x, y)=C_{m n} H_{m}\left(\sqrt{\frac{2 \pi}{L \lambda} x}\right) H_{n}\left(\sqrt{\frac{2 \pi}{L \lambda} y}\right) \mathrm{e}^{-\frac{x^{2}+y^{2}}{(L \lambda / \pi)}} .
$$

Here $C_{m n}$ is a constant associated with the parameters of the resonator cavity; $H_{m}$ and $H_{n}$ are mth-orderand nth-order Hermite polynomial, respectively:

*qhwang@njust.edu.cn; fax 025-84303073

14th Conference on Education and Training in Optics and Photonics: ETOP 2017, edited by Xu Liu,

Xi-Cheng Zhang, Proc. of SPIE Vol. 10452, 104525U · C 2017 ICO, IEEE, OSA, SPIE

CCC code: $0277-786 \mathrm{X} / 17 / \$ 18 \cdot$ doi: $10.1117 / 12.2269884$

Proc. of SPIE Vol. $10452104525 \mathrm{U}-1$ 


$$
H_{m}(X)=\sum_{k=0}^{\left[\frac{m}{2}\right\rfloor} \frac{(-1)^{k} m !}{k !(m-2 k) !}(2 X)^{m-2 k}, \quad(m=0,1,2, \cdots),
$$

where $\left[\frac{m}{2}\right]$ returns the integer portion of a number.

To a symmetric confocal resonator with cavity length $L$, Laser wavelength $\lambda$, and diameter $2 a$ round mirror, when the Fresnel number $N=\frac{a^{2}}{\lambda L}$ tends to infinity, the optical field can be described by the laguerre-Gaussian function ${ }^{[6]}$ :

$$
U_{m n}(r, \varphi)=C_{m n}\left(\sqrt{2} \frac{r}{\omega_{0 s}}\right)^{m} L_{n}^{m}\left(2 \frac{r^{2}}{\omega_{0 s}^{2}}\right) \mathrm{e}^{-\frac{r^{2}}{\omega_{0 s}^{2}}} \cos m \varphi .
$$

Here $C_{m n}$ is a constant associated with the parameters of the resonator cavity, $\omega_{0 s}=\sqrt{L \lambda / \pi}$, and $L_{n}^{m}$ is the generalized Laguerre polynomial of order $\mathrm{n}$ :

$$
L_{m}^{n}(\zeta)=\sum_{k=0}^{n} \frac{(n+m) !(-\zeta)^{k}}{(m+k) ! k !(n-k) !} .
$$

Equation (1) and (3) show that optical amplitudes of the basis modes $(m=0, n=0)$ obey the Gaussian distribution for both quadrate mirror pair and round ones. The amplitude of the basis mode decays from the center to the edge of the mirror. At the distance $r=\sqrt{L \lambda / \pi}$, the amplitude drops down to $1 / \mathrm{e}$ of the center, which is called the spot diameter of the basis mode.

When a series of integers $(m, n)$ are chosen, every high order modes can be obtained. For the square mirror, with the increase of $\mathrm{x}$ and $\mathrm{y}$, Gaussian function decays monotonously and Hermite polynomial is positive or negative alternately. The zero points of Hermite polynomial determine the nodal lines. The Gaussian function and Hermite polynomial jointly determine the profile of TEM. There are $\mathrm{m}$ zero points for $\mathrm{mth}$-order Hermite polynomial (i.e. there are $\mathrm{m}$ roots for $\left.H_{m}(X)=0\right)$, then $\mathrm{TEM}_{\mathrm{mn}}$ has $\mathrm{m}$ nodal lines along the direction of $\mathrm{x}$ axis and $\mathrm{n}$ nodal lines along the direction of $\mathrm{y}$ axis. For the round mirrors, the position and number of nodes along the radial direction is determined by generalized Laguerre polynomial and the nodes along the direction of argument $\varphi$ is determined by $\cos m \varphi$. TEM $\mathrm{mn}_{\mathrm{m}}$ of the round mirrors has $\mathrm{m}$ nodal lines along the direction of argument $\varphi$, and $\mathrm{n}$ nodal lines along the radial direction.

When the amplitude $\mathrm{U}$ of optical field is known, the light intensity distribution I on the mirror can be calculated using the relation $I=U \cdot U^{*}$.

Knowing the amplitude of optical field on the mirror, the optical field at any point of the confocal cavity can be calculated by Fresnel-Kirchhoff diffraction integral formula. For the square mirrors, Boyd and Gordon had proved that the field in the confocal cavity can be expressed as ${ }^{[5]}$

$$
E_{m n}(x, y, z)=A_{m n} E_{0} \frac{\omega_{0}}{\omega(z)} H_{m}\left(\frac{\sqrt{2}}{\omega(z)} x\right) H_{n}\left(\frac{\sqrt{2}}{\omega(z)} y\right) \mathrm{e}^{-\frac{r^{2}}{\omega^{2}(z)}} \mathrm{e}^{-i \varphi(x, y, z)},
$$

where the cavity center is selected as the coordinate origin, $\omega(z)=\omega_{0} \sqrt{1+\left(\frac{z}{f}\right)^{2}}, r=\sqrt{x^{2}+y^{2}}, \omega_{0}=\sqrt{L \lambda /(2 \pi)}$ is the waist radius of basis mode, and $f=L / 2$ is the focal length of the mirror. Similarly, the field inside the confocal cavity constructed by the round mirrors can be expressed as ${ }^{[6]}$

$$
E_{m n}(r, \varphi, z)=A_{m n} \frac{\omega_{0}}{\omega(z)}\left(\sqrt{2} \frac{r}{\omega(z)}\right)^{m} L_{n}^{m}\left(2 \frac{r^{2}}{\omega^{2}(z)}\right) \mathrm{e}^{-\frac{r^{2}}{\omega^{2}(z)}} \cos m \varphi \mathrm{e}^{-\frac{r^{2}}{\omega^{2}(z)}} \mathrm{e}^{-i \varphi(r, \varphi, z)}
$$

Given the transmission of the output mirror, Equation (5) and (6) can also work outside the confocal cavity. Because every stable cavity has an equivalent confocal cavity, Equations (1) - (6) have universal significance. 


\section{OPTICAL FIELD SIMULATION}

Based on Equations (1)-(6), we have computed the optical field and light distribution and drawn the profile of diffraction pattern with the help of Python. In this paper, we take the He-Ne Laser for example. During the simulation, the cavity length $L=1 \mathrm{~m}, \lambda=0.6328 \mu \mathrm{m}$.

\subsection{Light distribution on the mirror}

First of all, our program can draw the $3 \mathrm{~d}$ and $2 \mathrm{~d}$ profiles of light distribution on the mirror for arbitrary transverse modes number $\mathrm{m}$ and $\mathrm{n}$. Figure 1 shows the light distribution of $\mathrm{TEM}_{22}$ on the mirror for the symmetric confocal resonator constructed with quadrate mirrors. There are 2 nodal lines both along the direction of $\mathrm{x}$ axis and $\mathrm{y}$ axis, where the light intensity is zero. The characteristics of $\mathrm{TEM}_{22}$ are embodied in Figure 1. Figure 2 shows the light distribution of $\mathrm{TEM}_{13}$ on the mirror for the round mirrors. There are 1 nodal line along the direction of argument, and 3 nodal lines along the radial direction. Figure 2 clearly demonstrates the characteristics of $\mathrm{TEM}_{13}$.

(a)3d light distribution

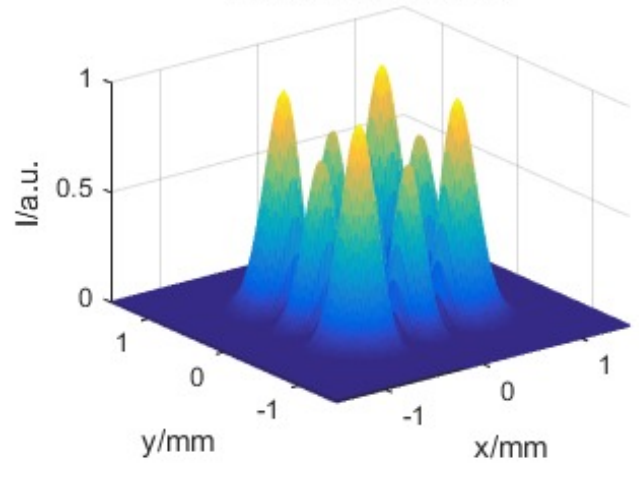

(b)2d light distribution

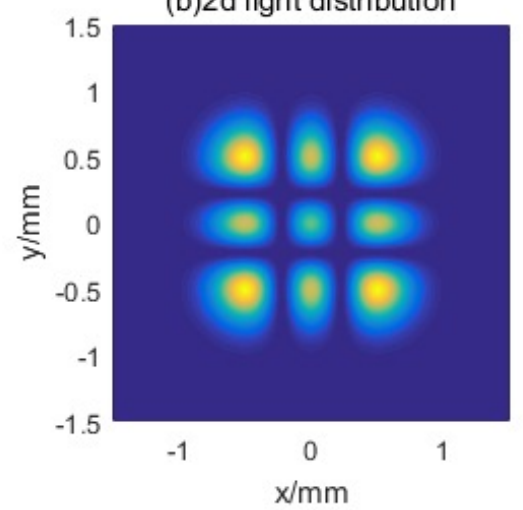

Fig.1 The light distribution of $\mathrm{TEM}_{22}$ for quadrate mirrors

(a)3d light distribution

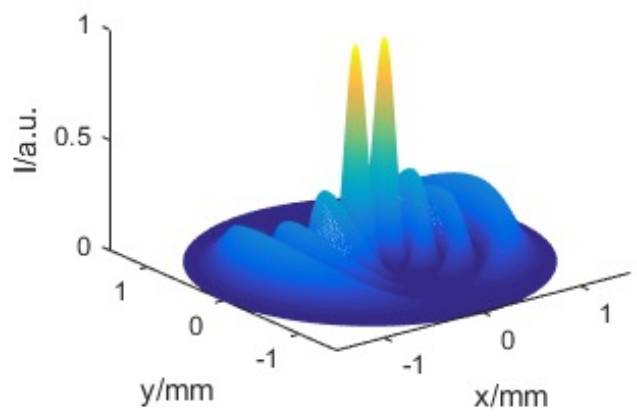

(b)2d light distribution

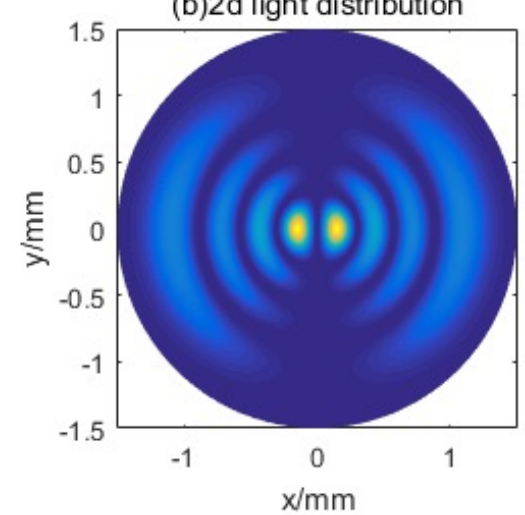

Fig.2 The light distribution of $\mathrm{TEM}_{13}$ for round mirrors

\subsection{Light distribution of the traveling wave}

We also simulated the light distribution of the traveling wave. Figure 3 shows the $\mathrm{TEM}_{31}$ mode for the quadrate mirrors. There are 3 nodal lines along the direction of $\mathrm{x}$ axis and 1 nodal line along the $\mathrm{y}$ direction. Figure 4 shows the TEM $\mathrm{T}_{31}$ mode for the round mirrors. There are 3 nodal lines along the direction of argument and 1 nodal line along the radial direction. Figure 3(a) and Figure 4(a) demonstrate the light distribution at the center of the cavity. Figure 3(b) and Figure 4(b) show the light distribution 0.5 meters apart from the center of the cavity. The light distributions on the mirrors (1 meter apart from the center of the cavity) are present in Figure 3(c) and Figure 4(c). Figure 3 and 4 perfectly show the features of the light distribution of the traveling wave. The spot size is smallest at the center of the cavity and increases with the increase of the distance from the center of the cavity. 

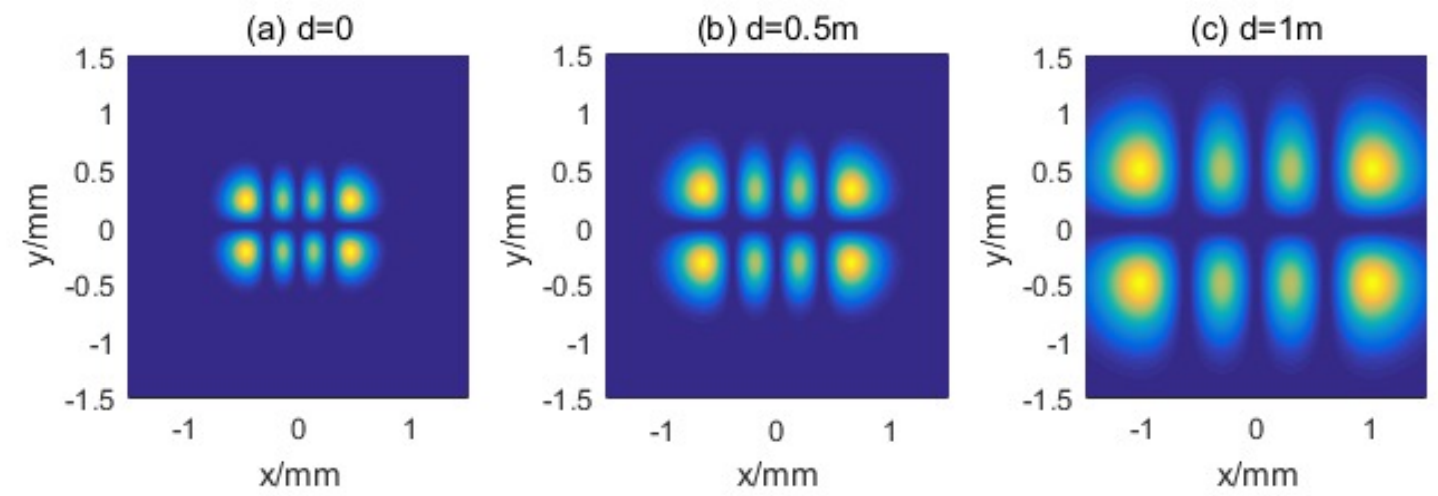

Fig.3 $\mathrm{TEM}_{31}$ mode for the quadrate mirrors
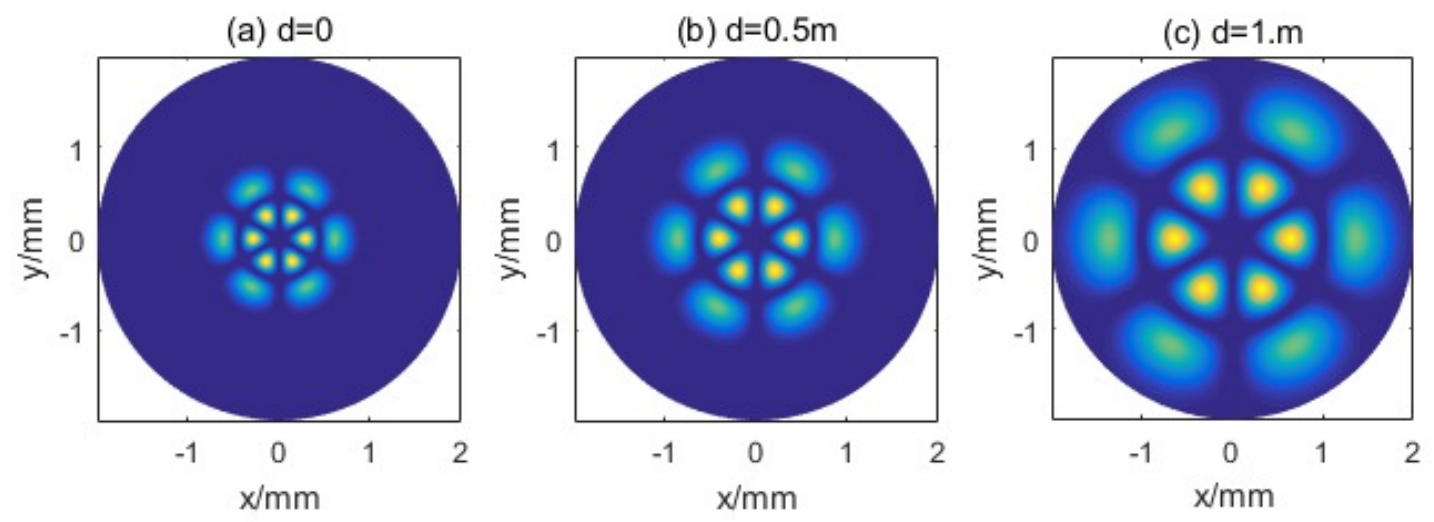

Fig. $4 \mathrm{TEM}_{31}$ mode for the round mirrors

\subsection{Light distribution of mixed modes on the mirror}

Multiple modes are also simulated in our program. $C_{m n}$ in Equations (1) and (3) is influenced by the structure and working material of the cavity and all kinds of loss. Usually we do not care for the value of $\mathrm{C}_{\mathrm{mn}}$ because we only need the relative value of the light distribution. In order to highlight the features of mixed modes, here $\mathrm{C}_{\mathrm{mn}}$ is set to be $0.6^{(m+n)}$ for square mirrors and $0.9^{(m+n)}$ for round mirrors. Figure 5 shows the diffraction patterns on the square mirrors for $\mathrm{TME}_{00}, \mathrm{TEM}_{11}$ and the mixture respectively. There is no nodal line in $\mathrm{TME}_{00}$ on the whole mirror and a nodal line in $\mathrm{TME}_{11}$ along the direction of $\mathrm{x}$ and $\mathrm{y}$, respectively. The mixed mode of $\mathrm{TME}_{00}$ and $\mathrm{TEM}_{11}$ presents a cross-like distribution, which agrees with theoretical analysis. Figure 6 shows the diffraction patterns on the round mirrors for $\mathrm{TME}_{00}, \mathrm{TEM}_{11}$ and the mixture respectively. $\mathrm{TME}_{00}$ has no nodal line on the whole mirror. $\mathrm{TME}_{11}$ has a nodal line along both the argument direction and the radial direction, respectively. The superposition of $\mathrm{TME}_{00}$ and $\mathrm{TEM}_{11}$ strengthens some part of the pattern, but the distribution characteristic can still be recognized. 
(a) $\mathrm{TEM}_{00}$

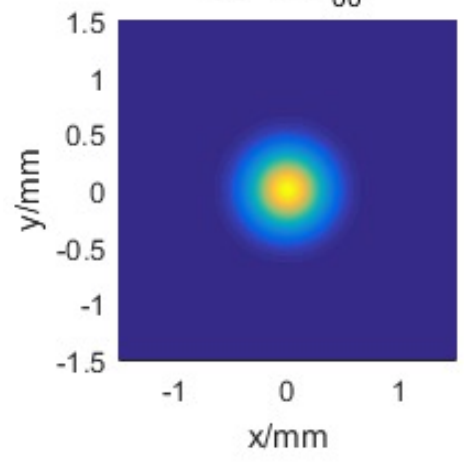

(b) TEM $_{11}$

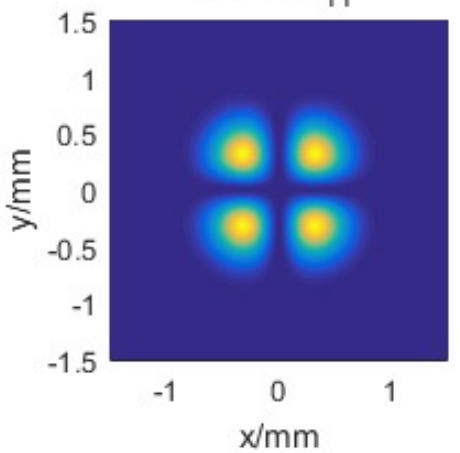

(c) $\mathrm{TEM}_{00}+\mathrm{TEM}_{11}$

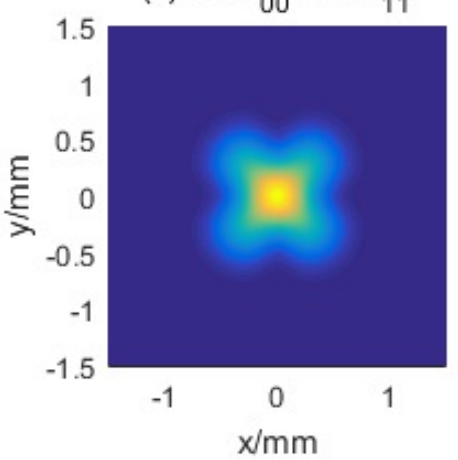

Fig. 5 Light distribution of mixed modes on the square mirror
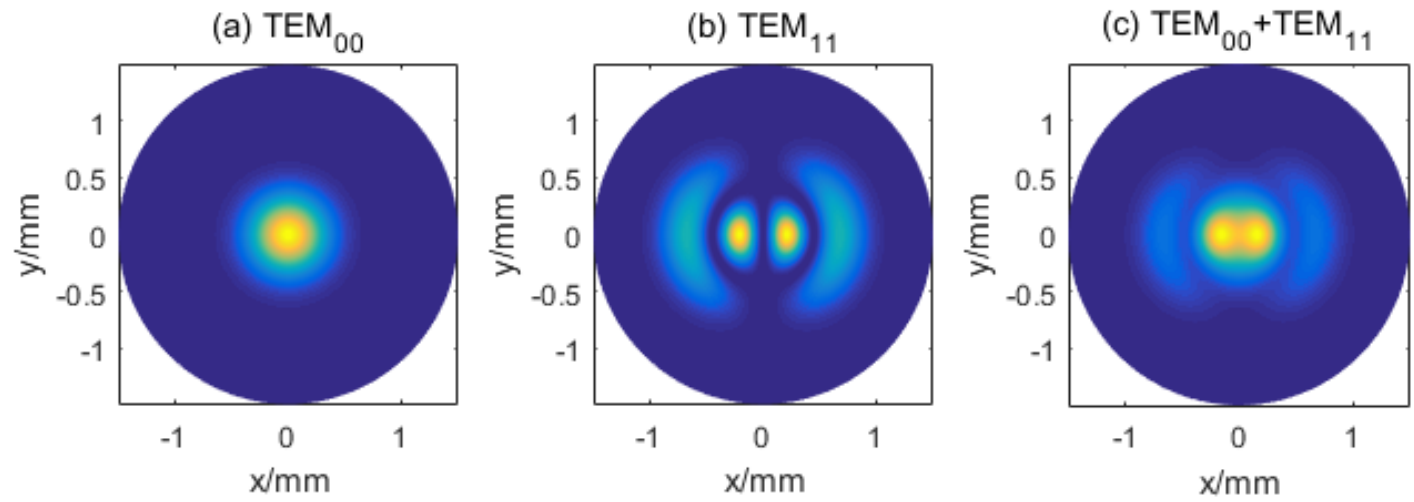

Fig.6 Light distribution of mixed modes on the round mirror

\section{CONCLUSION}

We have developed a suite of Python programs to simulate the transverse modes of the symmetric confocal resonator. Light patterns on the mirror, or inside the cavity can be visualized. The mixed modes can also be demonstrated intuitively. Pseudocolor images are used to show the relative intensity, which is more distinct than gray scale ones. During teaching the course of laser principle, our Python programs have improved the students' interest in learning and helped students understand the pattern distribution of laser resonator.

\section{REFERENCES}

[1] Siegman, A.E., [Lasers], University Science Books, California, 744-759 (1986).

[2] The Python Software Foundation, “About the Python,"https://www.python.org/about/(Mar 22 2017)

[3] SciPy developers, "Special functionsh, "https://docs.scipy.org/doc/scipy/reference/special.html (Mar 09 2017)

[4] Fox, A. G., Li,T., "Resonant modes in a maser Interferometer," Bell. Syst Tech. J., 40(3), 453-488 (1961).

[5] Svelto, O., [Principles of Lasers], Springer, New York, 158-161 (2010).

[6] Allen ,L., Beijersbergen,M. W., Spreeuw R. J. C. and Woerdman, J. P., "Orbital angular momentum of light and the transformation of Laguerre-Gaussian laser modes," Physical Review A., 45 (11), 8185-8189 (1992). 